\title{
Division-Interpolation methods and Nullstellensätze
}

\author{
Carlos A. Berenstein and Alain Yger \\ To Leon Ehrenpreis, friend and teacher
}

\section{Introduction}

The Fundamental Principle of Ehrenpreis asserts that any solution (in an appropiate functional space, which we will assume, to simplify, is the space of $C^{\infty}$ functions in $\mathbf{R}^{n}$ ) of a system of homogeneous linear partial differential equations with constant coefficients in $\mathbf{R}^{n}, n \geq 2$, can be can be represented in terms of the exponential polynomials solutions of the system [Ehr, Pal, Bjo]. Another way to phrase the Fundamental Principle is to say that in certain spaces of entire functions with restricted growth in $\mathbf{C}^{n}$ one has an explicit linear (and continuous) division algorithm with remainder with respect to the ideal generated by the symbols of these equations. Such an assertion, even the fact the exponential polynomial solutions of the system are dense in the space of all solutions, fails for systems of homogeneous convolution equations $\mu_{1} * f=\cdots=\mu_{m} * f=0[\mathrm{Gu}]$. Moreover, there are few positive examples of transcendental nature where we know that the Fundamental Principle (or just Spectral Synthesis) holds. Note that it is still possible to state this principle by saying that in the Paley-Wiener algebra there is an explicit linear and continuous division algorithm with remainder with respect to the ideal generated by the Fourier transforms $\hat{\mu}_{j}$ of the convolution operators. In particular, the ideal generated by these Fourier transforms should be closed. By extension, we will say that a system of convolution equations

$$
\mu_{1} * f=\ldots=\mu_{m} * f=0
$$

1991 Mathematics Subject Classification. Primary 14Q20, 32A37. Secondary 11J99.

This research has been partially supported by NSA and NSF grants. 
such that the spectrum $\left\{z \in \mathbf{C}^{n}, \hat{\mu}_{j}(z)=0, j=1, \ldots, m\right\}$ is empty (the system is inconsistent) satisfies the Fundamental Principle if and only if the ideal generated by the $\hat{\mu}_{j}$ in the Paley-Wiener algebra is a non proper ideal; this is equivalent to say that there exists an analytic Bézout (or deconvolution) identity

$$
\delta=\sum_{j=1}^{m} \nu_{j} * \mu_{j}
$$

where the deconvolutors $\nu_{j}$ are distributions with compact support, or, which is equivalent, a global Lojasiewicz type inequality

$$
\sum_{j=1}^{m}\left|\hat{\mu}_{j}(z)\right| \geq \gamma \frac{e^{-C\|I m z\|}}{(1+\|z\|)^{N}}, \gamma>0, C \geq 0, N \in \mathbf{N}
$$

A natural class of systems of convolution equations to consider is that of systems of homogeneous linear difference-differential equations with constant coefficients and delays in a discrete subgroup $\Lambda \subset \mathbf{R}^{n}$ with rank $p$; when the equations are just difference equations, such examples are of transcentental nature if the rank of $\Lambda$ is strictly bigger than $n$; otherwise, any value of $p \geq 1$ leads to examples that cannot be reduced to algebraic ones (for example, the simplest case is $p=1$, which has been extensively studied in [BY6]). In any case, if the $\mu_{j}$ are such differencedifferential equations, the $\hat{\mu}_{j}$ are exponential polynomials with frequencies in $i \Lambda$. For $n=2$ the Fundamental Principle for this kind of systems holds when there are exactly one or two equations [BTY]. Within this class of systems, if $n=1,2$ and the number of equations is strictly bigger than $n$, or when $n \geq 3$, there are examples where the ideal generated by the $\hat{\mu}_{j}$ in the Paley-Wiener algebra is not closed. If the corresponding spectrum is non-empty then the Fundamental Principle fails. This is also true if we extend the concept of Fundamental Principle to inconsistent systems as we did before. We do not know what happens for the Spectral Synthesis. These examples are related to the transcendental nature of $\Lambda$ [BY1]. An interesting example for $n=1, m=2$, due to Ehrenpreis, occurs when $\hat{\mu}_{2}(z)=\frac{d}{d z} \hat{\mu}_{1}(z)$ and the two Fourier transforms have no common zeros. If the ideal were closed, the zeros of $\hat{\mu}_{1}$ have to be well-separated, that is, there are constants $c_{1}, c_{2}, d>0$ such that if $z, z^{\prime}$ are two distinct zeros of $\hat{\mu}_{1}$ then

$$
\left|z-z^{\prime}\right| \geq c_{1} \frac{\exp \left(-c_{2}|\operatorname{Im} z|\right)}{(1+|z|)^{d}}
$$

This separation condition is not satisfied in general, e.g.,

$$
\hat{\mu}_{1}(z)=\sin z \sin (\alpha z),
$$

where $\alpha$ is a Liouville number. There is also an example due to Selberg where the frequencies are algebraic but the separation (1.3) is not satisfied because the coefficients of the exponentials are not algebraic [BY2]. Ehrenpreis has conjectured that the separation condition is satisfied for the zeros of any exponential polynomial with purely imaginary algebraic frequencies and algebraic coefficients. In this setting, this conjecture appears as a particular case of the following: any system 
of linear difference-differential operators with frequencies in $\overline{\mathbf{Q}}^{n}$ and coefficients in $\overline{\mathbf{Q}}$ satisfies the Fundamental Principle. For example, to test the Ehrenpreis's Conjecture when

$$
\begin{aligned}
& \mu_{1}(t)=\sum_{p=\left(p_{0}, \ldots, p_{n-1} \in \mathbf{Z}^{n}\right.} a_{p}^{(1)}(D) * \delta\left(t-\left(p_{0}+p_{1} \omega_{1}+\ldots+p_{n-1} \omega_{n-1}\right)\right), t \in \mathbf{R} \\
& \mu_{2}(t)=\sum_{p=\left(p_{0}, \ldots, p_{n-1}\right) \in \mathbf{Z}^{n}} a_{p}^{(2)}(D) * \delta\left(t-\left(p_{0}+p_{1} \omega_{1}+\ldots+p_{n-1} \omega_{n-1}\right)\right), t \in \mathbf{R},
\end{aligned}
$$

where $n$ is now the rank of the subgroup $\Lambda$ generated by the $\mathbf{Q}$-linearly independent algebraic numbers $1, \omega_{1}, \ldots, \omega_{n-1}$, one could check if the system of $n+1$ equations in $\mathbf{R}^{n}$

$$
\begin{aligned}
\frac{\partial f}{\partial z_{2}}-\omega_{1} \frac{\partial f}{\partial z_{1}}=0, \ldots, \frac{\partial f}{\partial z_{n}}-\omega_{n-1} \frac{\partial f}{\partial z_{n-1}} & =0 \\
\sum_{p_{0}, \ldots, p_{n-1} \in \mathbf{Z}^{n}} a_{p}^{(1)}\left(\frac{\partial}{\partial z_{1}}\right) f\left(t_{1}-p_{0}, \ldots, t_{n}-p_{n-1}\right) & =0 \\
\sum_{p_{0}, \ldots, p_{n-1} \in \mathbf{Z}^{n}} a_{p}^{(2)}\left(\frac{\partial}{\partial z_{1}}\right) f\left(t_{1}-p_{0}, \ldots, t_{n}-p_{n-1}\right) & =0
\end{aligned}
$$

satisfies the Fundamental Principle. We have a system of difference-differential equations in $n$ variables with delays in $\mathbf{Z}^{n}$. When $\mu_{1}$ is a difference operator then the two last equations in (1.2) are difference equations with integral delays.

There are two ways to try to verify that the Fundamental Principle holds:

- One is to study the geometry of the connected components of the sets where the $\hat{\mu}_{j}$ are simultaneously small, and then apply in such components DivisionInterpolation formulas that extend to the analytic setting classical formulas given by Lagrange, Jacobi, Kronecker in the case of polynomials. Under natural hypotheses, there is an explicit division algorithm with remainder, found using cohomology with bounds [BT]. The use of such a method is restricted to the case where the $\hat{\mu}_{j}$ define a complete intersection variety. We will see in Section 2 how these Division-Interpolation formulas are also essential to get explicit versions of the algebraic Nullstellensatz. It is even possible to adapt the analytic formalism to study systems of polynomial equations over a field of positive characteristic, a local ring, or an integral domain.

- The other method is based on the use of weighted integral representation formulas of the Bochner-Martinelli type; such formulas (see for example [BGVY] or [BY6]) provide explicit formulations of the Briançon-Skoda theorem [BS], the ideal being the ideal generated by the $\hat{\mu}_{j}$ in the space of entire functions in $n$ complex variables, and lead to division algorithms of the form

$$
h(z)=\sum_{j=1}^{m} T_{j} h(z) \hat{\mu}_{j}(z)+T_{0} h(z)
$$

with

$$
T_{j} h(z)=\sum_{s}<h T_{j s}(\zeta), \omega_{j s}(\zeta, z)>, j=0, \ldots, m
$$


where the $T_{j s}$ are currents (linear combinations of currents of the form PrincipalValue distribution times Residue current, in the sense of $[\mathrm{CH}])$, the $\omega_{j s}$ are smooth forms in $\zeta$ that depend holomorphically of $z$ and decrease (in $\zeta$ ) at infinity in such a way that the duality brackets

$$
<h T_{j s}(\zeta), \omega_{j s}(\zeta, z)>
$$

are well defined when $h$ is in the Paley-Wiener algebra and remain (as functions of $z$ ) in this algebra. Moreover, in the complete intersection case, one has

$$
T_{0} h(z)=<h \bar{\partial} \frac{1}{\hat{\mu}_{1}(\zeta)} \wedge \cdots \wedge \bar{\partial} \frac{1}{\hat{\mu}_{m}(\zeta)}, \omega_{0}(\zeta, z)>
$$

where $\bar{\partial} \frac{1}{\hat{\mu}_{1}(\zeta)} \wedge \cdots \wedge \bar{\partial} \frac{1}{\hat{\mu}_{m}(\zeta)}$ is the residue current introduced by Coleff-Herrera $[\mathrm{CH}]$. This current is known to be annihilated locally by the ideal generated by the $\hat{\mu}_{j}$, so that (1.5), when it holds, provides a division algorithm with remainder. In the general case, all currents $T_{0 s}$ are locally annihilated by holomorphic functions whose germs near any point $z$ in $\mathbf{C}^{n}$ belong to the integral closure (in $\mathcal{O}_{z}$ ) of the $\min (n, m)$-th power of the ideal generated by the germs at $z$ of the $\hat{\mu}_{j}, j=1, \ldots, m$, and by antiholomorphic functions that vanish on the set of common zeroes of the $\hat{\mu}_{j}$. Such currents are supported by this set and one can view also (1.5) in the general case as a Division-Interpolation formula, even if it does not provide in any obvious way a division algorithm with remainder. In any case, the difficulty that arises when one wants to write such division formulas is to control the growth at infinity of the distributions that appear as coefficients of the $T_{j s}, j=0, \ldots, m$. Such coefficients can be recovered from the study of the meromorphic multivariate distribution-valued Gamma function

$$
\left(s_{1}, \ldots, s_{m}\right) \mapsto\left[\varphi \mapsto \int_{\mathbf{C}^{n}}\left|\hat{\mu}_{1}\right|^{2\left(s_{1}-1\right)} \cdots\left|\hat{\mu}_{m}\right|^{2\left(s_{m}-1\right)} \varphi d \bar{\zeta} \wedge d \zeta\right] .
$$

This function is a meromorphic function whose polar set is a union of hyperplanes

$$
q_{1} s_{1}+\ldots+q_{m} s_{m}+q_{0}=0, q_{1}, \ldots, q_{m} \in \mathbf{N}, q_{0} \in \mathbf{Z} .
$$

The action of any distribution which appears as a coefficient of a current $T_{j s}$ on a test function equals a residue of this meromorphic Gamma function, respect to some set of $n$ polar divisors corresponding to hyperplanes with independent directions. For example, to prove the Ehrenpreis Conjecture when

$$
\mu_{1}(t)=\sum_{p=\left(p_{0}, \ldots, p_{n-1)} \in \mathbf{Z}^{n}\right.} a_{p}^{(1)} \delta\left(t-\left(p_{0}+p_{1} \omega_{1}+\ldots+p_{n-1} \omega_{n-1}\right)\right),
$$

where the $a_{p}^{(1)}$ are algebraic numbers (that is for exponential sums instead of exponential polynomials), it would be enough to study the meromorphic continuation of a distribution-valued function of the form

$$
\begin{gathered}
\left(s_{1}, \ldots, s_{n-1}, t_{1}, t_{2}\right) \mapsto \\
{\left[\varphi \mapsto \int_{\mathbf{C}^{n}}\left(\prod_{j=1}^{n-1}\left|\zeta_{j+1}-\omega_{j} \zeta_{1}\right|^{2\left(s_{j}-1\right)}\right)\left|P_{1}\left(e^{-i \zeta}\right)\right|^{2\left(t_{1}-1\right)}\left|P_{2}\left(e^{-i \zeta}\right)\right|^{2\left(t_{2}-1\right)} \varphi d \bar{\zeta} \wedge d \zeta\right]}
\end{gathered}
$$


where the polynomials $P_{j} \in \overline{\mathbf{Q}}\left[X_{0}, \ldots, X_{n-1}\right]$ are given by

$$
P_{j}\left(X_{0}, \ldots, X_{n-1}\right):=\sum_{p} a_{p}^{(j)} X_{0}^{p_{0}} \cdots X_{n-1}^{p_{n-1}}, j=1,2 .
$$

Though we still do not know how to handle this question, it seems important to notice that the two functions $P_{l}\left(e^{-i \zeta_{1}}, \ldots, e^{-i \zeta_{n}}\right), l=1,2$, are periodic with periods $2 \pi e_{j}, j=1, \ldots, n$, where the vectors $e_{j}$ form the canonical basis of $\mathbf{R}^{n}$. On the other hand, by Baker's theorem [Ba], any linear combination

$$
\log \alpha-\omega \log \beta+2 \pi\left(k_{1}+k_{2} \omega\right), \alpha, \beta, \omega \in \overline{\mathbf{Q}} \backslash \mathbf{Q}, k_{1}, k_{2} \in \mathbf{Z}
$$

which is not zero satisfies

$$
\left|\log \alpha-\omega \log \beta+2 \pi\left(k_{1}+k_{2} \omega\right)\right| \geq \gamma(\alpha, \beta, \omega)\left(1+\left|k_{1}\right|+\left|k_{2}\right|\right)^{-C(\alpha, \beta, \omega)} .
$$

Therefore, it would be useful to have a semi-local resolution of singularities (in a compact domain of the form $\max _{j}\left|\operatorname{Re} \zeta_{j}\right| \leq 2 \pi, \max _{j}\left|\operatorname{Im} \zeta_{j}\right| \leq T$ ) for the collection of hypersurfaces

$$
\left(\prod_{j=1}^{n-1}\left(\zeta_{j+1}-\omega_{j} \zeta_{1}+2 \pi\left(k_{j+1}-\omega_{j} k_{1}\right)\right) P_{1}\left(e^{-i \zeta}\right) P_{2}\left(e^{-i \zeta}\right)=0, k_{1}, \ldots, k_{n} \in \mathbf{Z}^{n}\right.
$$

such that the centers of the balls at any step in the succesive blow-ups could be chosen to have coordinates in $\log \overline{\mathbf{Q}}$. It would seem that under these conditions one could control the growth of the distributions that appear as residues of the meromorphic function (1.7) with respect to polar divisors and, thus, prove the Ehrenpreis Conjecture for sums of exponentials. For the moment, the only case which is completely settled is the case where the rank of $\Lambda$ is 2 . There are also results about the separation of zeros when $\Lambda$ is generated by $1, \omega, \omega^{2}$, where $\omega$ is a cubic irrational [BY2], [BY6].

The analytic Division-Interpolation methods mentioned above can be used to prove effective versions of Hilbert's Nullstellensatz over a field of characteristic zero [BY8, BY9]. We will see in the next section that some of these analytic tools have algebraic counterparts. For instance, the Lagrange-Kronecker interpolation formula, the residue symbols, and the Lipman-Teissier theorem (which corresponds to the Briançon-Skoda theorem) [LT], [LS]. In that section we will explain how they can be used to prove effective Nullstellensätze over fields of arbitrary characteristic.

\section{An arithmetic version of the algebraic Nullstellensatz}

Let $\mathbf{K}$ be an algebraically closed field (of arbitrary characteristic) equipped with an absolute value and $P_{1}, \ldots, P_{n}$ be polynomials in $\mathbf{K}\left[x_{1}, \ldots, x_{n}\right]$ of respective degrees $D_{1}, \ldots, D_{n}$, defining a discrete, hence finite, algebraic variety in $\mathbf{K}^{n}$. One can see, as in [Ko], that there exist constants $c>0$ and $K \geq 0$ such that for any $x,|x| \geq K$ one has

$$
\max _{1 \leq j \leq n} \frac{\left|P_{j}(x)\right|}{|x|^{D_{j}}} \geq c|x|^{-D_{1} \cdots D_{n}}
$$


When the $P_{j}$ have no common zero at infinity, one can replace $D_{1} \cdots D_{n}$ by 0 . When the polynomial map is proper, that is, when $\mathbf{K}\left[x_{1}, \ldots, x_{n}\right]$ is a $\mathbf{K}\left[P_{1}, \ldots, P_{n}\right]$ finitely generated module, one has an inequality of the form

$$
\max _{1 \leq j \leq n} \frac{\left|P_{j}(x)\right|}{|x|^{\delta_{j}}} \geq c,|x| \geq K,
$$

where $0<\delta_{j} \leq D_{j}, 1 \leq j \leq n$. The inequality (2.1) can be checked easily when one considers the homogeneous polynomials $\mathcal{P}_{j}, j=1, \ldots, n$ and the functions $p_{1}(x), \ldots, p_{n}(x)$ which correspond to the functions $\mathcal{P}_{j}(x)$ expressed in affine coordinates near some point of the hyperplane $x_{0}=0$. Let $\pi: Z \mapsto \mathbf{C}^{n}$ be the normalized blow-up of the ideal generated by the $p_{j}$. It follows from Bézout's theorem that at least one $p_{j} \circ \pi$ vanishes to an order strictly smaller than $D_{1} \ldots D_{n}$ along each component of the exceptional divisor of the normalized blow-up ([Ko, Te1]). In fact, as it was pointed to us by M. Hickel, a careful analysis of the proof in [Ko] shows that (2.1) can be improved in some cases, the exponent $D_{1} \cdots D_{n}$ can be reduced to a value $\Delta$ expressible in terms of the invariants introduced in [Te2].

Assume that $Q$ belongs to the ideal $I$ generated by the $P_{j}$ and let $\mathcal{Q}$ be the homogenization of $Q$. It follows from (2.1) that the product $\mathcal{Q} x_{0}^{D_{1} \cdots D_{n}}$, considered as a formal power series in $\mathbf{K}\left[\left[x_{0}, \ldots, x_{n}\right]\right]$, is in the integral closure $\overline{\mathcal{I}}$ of the ideal $\mathcal{I}$ generated in $\mathbf{K}\left[\left[x_{0}, \ldots, x_{n}\right]\right]$ by the $\mathcal{P}_{j}$. One knows now from the Lipman-Teissier theorem that

$$
\overline{\mathcal{I}}^{l} \subseteq \overline{\mathcal{I}}^{l} \subseteq \mathcal{I},
$$

where $l \leq n$ is the minimal number of elements in $\mathcal{I}$ which generate a reduction of this homogeneous ideal. Therefore, we have $x_{0}^{l D_{1} \cdots D_{n}} \mathcal{Q}^{l} \in \mathcal{I}$. If now we go back to the affine coordinates, we obtain that

$$
Q^{l}=\sum_{k=1}^{n} A_{k} P_{k}, \operatorname{deg} A_{k} P_{k} \leq l\left(\operatorname{deg} Q+D_{1} \cdots D_{n}\right) .
$$

Whenever we can replace $D_{1} \ldots D_{n}$ by $\Delta<D_{1} \cdots D_{n}$ in (2.1), we obtain

$$
Q^{l}=\sum_{k=1}^{n} A_{k} P_{k}, \operatorname{deg} A_{k} P_{k} \leq l(\operatorname{deg} Q+\Delta) .
$$

The product $l \Delta$ is certainly bounded by $n D_{1} \cdots D_{n}$, which is not a very sharp bound in general. If $Q$ belongs to the radical of the ideal and $\nu$ is the maximum of the Noether exponents at all the common zeros of the $P_{j}$, one has

$$
Q^{\nu l}=\sum_{k=1}^{n} A_{k} P_{k}, \operatorname{deg} A_{k} P_{k} \leq l(\nu \operatorname{deg} Q+\Delta) .
$$

On the other hand, using a totally different method, based on cohomology with supports, which bypasses the use of the Lojasiewicz inequality (2.1), Kollár (also in [Ko]) was able to prove that if $Q$ is in the radical of the ideal $I$, then, one has

$$
Q^{e}=\sum_{k=1}^{n} A_{k} P_{k}, \max \left(e, \operatorname{deg}\left(A_{k} P_{k}\right)\right) \leq \prod_{j=1}^{n} \max \left(D_{j}, 3\right) .
$$


Such a result holds for ideals generated by an arbitrary number $m$ of generators by replacing $n$ by $\min \{m, n\}$ and taking the degrees in decreasing order. Note that $\nu l \leq \nu n$ depends only on the geometric information in the affine space, while the exponent $e$ is estimated in terms of the decomposition of the homogeneous ideal $\mathcal{I}$.

Such results give only degree bounds for the algebraic Nullstellensatz. In order to deal with these problems from the arithmetic point of view (for example when the polynomials are in $\mathbf{A}\left[x_{1}, \ldots, x_{n}\right]$, where $\mathbf{A}$ is a factorial regular integral domain equipped with a logarithmic size such as $\mathbf{Z}$ or $\mathbf{F}_{p}\left[\tau_{1}, \ldots, \tau_{q}\right]$, or a regular local ring equipped with a valuation), it is essential to solve explicitly the identities (2.3) or (2.4), and, more generally, the Bézout problem: given $m$ polynomials $P_{1}, \ldots P_{m}$ in $\mathbf{A}\left[x_{1}, \ldots, x_{n}\right]$ without common zeroes in $\overline{\mathbf{K}}^{n}$, where $\mathbf{K}$ is the fraction field of the domain $\mathbf{A}$, find explicitly $a \in \mathbf{A}, A_{1}, \ldots, A_{m} \in \mathbf{A}\left[x_{1}, \ldots, x_{n}\right]$, with good degree and size estimates (in terms of the degree and size estimates of the data $\left.P_{j}, j=1, \ldots, m\right)$ such that

$$
a=\sum_{k=1}^{m} A_{k} P_{k}
$$

When $\mathbf{A}=\mathbf{Z}$ and the logarithmic size is the Mahler measure

$$
h(P)=\frac{1}{(2 \pi)^{n}} \int_{[0,2 \pi]^{n}} \log \left|P\left(e^{i \theta_{1}}, \ldots, e^{i \theta_{n}}\right)\right| d \theta_{1} \ldots d \theta_{n},
$$

the optimal result one could hope (in view of the arithmetic Bézout theorem that comes from multidimensional Arakelov theory [BGS]) would be that (2.5) holds with the additional estimates

$$
h(a)=\log |a| \leq \kappa(n) h D^{n+1} \text { and }\left\{\begin{array}{l}
\operatorname{deg}\left(A_{k} P_{k}\right) \leq \kappa(n) D^{n} \\
h\left(A_{k}\right) \leq \kappa(n) D^{n}(h+\log m+D)
\end{array}\right.
$$

where $D$ (resp. $h$ ) denotes the maximum of the degrees (resp. sizes) of the data $P_{j}$, $j=1, \ldots, m$. In fact, the Division-Interpolation formulas (introduced originally to solve the type of problems we mentioned in the introduction) give a possibility to solve explicitly the algebraic Nullstellensatz (though the estimates we get still fail to fit exactly with the ideal ones (2.6)). The key tool to do that, by analogy with our previous analytic work [BGVY], is to develop an operational multivariate Residue Calculus. Let us recall here the main points.

The local residue symbol, in the local $\operatorname{ring}{ }_{n} \mathcal{O}$, is defined by

$$
\operatorname{Res}\left[\begin{array}{c}
r_{0} d r_{1} \wedge \cdots \wedge d r_{n} \\
f_{1}, \ldots, f_{n}
\end{array}\right]=\lim _{\epsilon \mapsto 0} \gamma_{n} \int_{\|\zeta\|=\epsilon} \frac{r_{0}\left(\sum_{k=1}^{n}(-1)^{k-1} \overline{f_{k}} \bigwedge_{\substack{l=1 \\
l \neq k}}^{n} \overline{d f_{l}}\right) \wedge \bigwedge_{k=1}^{n} d r_{k}}{\left(\left|f_{1}\right|^{2}+\cdots+\left|f_{n}\right|^{2}\right)^{n}},
$$

whenever $r_{0}, \ldots, r_{n}, f_{1}, \ldots, f_{n}$ are elements in ${ }_{n} \mathcal{O}$ such that $\left(f_{1}, \ldots, f_{n}\right)$ defines a regular sequence and $\gamma_{n}=(-1)^{\frac{n(n-1)}{2}}(n-1) ! /(2 i \pi)^{n}$. It appears as a particular 
realization of a much more general algebraic concept. When $\mathcal{R}$ is a commutative $\mathbf{A}$ algebra, and $f_{1}, \ldots, f_{n}$ is a quasi-regular sequence in $\mathcal{R}$ such that the quotient $\mathbf{P}=$ $\mathcal{R} /\left(f_{1}, \ldots, f_{n}\right)$ is a finitely generated projective $\mathbf{A}$-module, the residue symbols

$$
\operatorname{Res}\left[\begin{array}{l}
r_{0} d r_{1} \wedge \cdots \wedge d r_{n} \\
f_{1}^{q_{1}+1}, \ldots, f_{n}^{q_{n}+1}
\end{array}\right], r_{0}, \ldots, r_{n} \in \mathbf{R}, q_{1}, \ldots, q_{n} \in \mathbf{N},
$$

can be defined as traces of particular operators in $\operatorname{Hom}_{\mathbf{A}}(\mathbf{P}, \mathbf{P})$. We refer to Lipman [L], chapter 3, for a presentation of this theory. The advantage of Lipman's approach (compared to the other algebraic approaches proposed for example by Scheja-Storch [ScS], Kunz [Ku1], see also [ElM1] for an overview of the subject) is that the theory is developped over an $\mathbf{A}$-commutative algebra instead of a $\mathbf{K}$-vector field, which makes it more attractive (even if we do not use its whole strenght here) if one thinks about division or interpolation problems from the arithmetic point of view.

The main tools that make Residue Calculus useful when solving effectivity problems are the diverse variants of the Transformation Law: when $f_{1}, \ldots, f_{n}$ and $g_{1}, \ldots, g_{n}$ are two quasi-regular sequences in $\mathcal{R}$ such that the quotients rings $\mathcal{R} /\left(f_{1}, \ldots, f_{n}\right)$ and $\mathcal{R} /\left(g_{1}, \ldots, g_{n}\right)$ are finitely generated projective $\mathbf{A}$-modules and

$$
g_{j}=\sum_{k=1}^{n} a_{j k} f_{k}, j=1, \ldots, n
$$

then, for any $r_{0}, \ldots, r_{n} \in \mathcal{R}$,

$$
\operatorname{Res}\left[\begin{array}{c}
r_{0} d r_{1} \wedge \cdots \wedge d r_{n} \\
f_{1}, \ldots, f_{n}
\end{array}\right]=\operatorname{Res}\left[\begin{array}{c}
r_{0} \operatorname{det}\left[a_{j k}\right] d r_{1} \wedge \cdots \wedge d r_{n} \\
g_{1}, \ldots, g_{n}
\end{array}\right] \text {. }
$$

A variant of this classical law (which can be found in [BY9]) is the following: suppose that $f_{0}, \ldots, f_{n}$ and $g_{1}, \ldots, g_{n}$ are $2 n+1$ elements in $\mathcal{R}$ such that the two sequences $\left(f_{0}, f_{1}, \ldots, f_{n}\right)$ and $\left(f_{0}, g_{1}, \ldots, g_{n}\right)$ are quasi-regular and the quotients $\mathcal{R} /\left(f_{0}, f_{1}, \ldots, f_{n}\right)$ and $\mathcal{R} /\left(f_{0}, g_{1}, \ldots, g_{n}\right)$ are finitely generated projective A-modules. Then, if there are positive integers $s_{1}, \ldots, s_{n}$ and elements $a_{j k} \in \mathcal{R}$ such that

$$
f_{0}^{s_{j}} g_{j}=\sum_{k=1}^{n} a_{j k} f_{k}, j=1, \ldots, n,
$$

one has, for any $q_{0} \in \mathbf{N}$, for any $r_{0}, r_{1}, \ldots, r_{n+1} \in \mathcal{R}$,

$$
\operatorname{Res}\left[\begin{array}{c}
r_{0} d r_{1} \wedge \cdots \wedge d r_{n+1} \\
f_{0}^{q_{0}+1}, f_{1}, \ldots, f_{n}
\end{array}\right]=\operatorname{Res}\left[\begin{array}{c}
r_{0} \operatorname{det}\left[a_{j k}\right] d r_{1} \wedge \cdots \wedge d r_{n+1} \\
f_{0}^{q_{0}+s_{1}+\ldots+s_{n}+1}, g_{1}, \ldots, g_{n}
\end{array}\right] \text {. }
$$

Among the diverse integral formulas that could provide at the same time division and interpolation in the analytic context, one of the most tractable from the algebraic point of view is the Cauchy-Weil's formula. If $\left(f_{1}, \ldots, f_{n}\right)$ is a regular sequence in ${ }_{n} \mathcal{O}$, and $g_{j k}, 1 \leq j, k \leq n$ are elements in ${ }_{2 n} \mathcal{O}$ such that

$$
f_{j}(z)-f_{j}(\zeta)=\sum_{k=1}^{n} g_{j k}(z, \zeta)\left(z_{k}-\zeta_{k}\right), j=1, \ldots, n,
$$


that is

$$
1 \otimes f_{j}-f_{j} \otimes 1=\sum_{k=1}^{n} g_{j k}\left(1 \otimes \zeta_{k}-\zeta_{k} \otimes 1\right), j=1, \ldots, n,
$$

then, for any $h$ in ${ }_{n} \mathcal{O}$, the following identity, between $h$ and the convergent power series below, holds:

$$
h(z)=\sum_{q=\left(q_{1}, \ldots, q_{n}\right) \in \mathbf{N}^{n}} \operatorname{Res}\left[\begin{array}{c}
h \operatorname{det}\left[g_{j k}(\cdot, z)\right] d \zeta_{1} \wedge \cdots \wedge d \zeta_{n} \\
f_{1}^{q_{1}+1}, \ldots, f_{n}^{q_{n}+1}
\end{array}\right] f_{1}^{q_{1}}(z) \cdots f_{n}^{q_{n}}(z) .
$$

Note that this is not the usual formulation of the Cauchy-Weil's formula as an integral representation formula, as it was extensively used in the semilocal context in $[\mathrm{BT}]$, that is

$$
h(z)=\frac{1}{(2 i \pi)^{n}} \int_{\Gamma_{f}(\epsilon)} \frac{h(\zeta) \operatorname{det}\left[g_{j k}(z, \zeta)\right] d \zeta_{1} \wedge \cdots \wedge d \zeta_{n}}{\prod_{k=1}^{n}\left(f_{k}(\zeta)-f_{k}(z)\right)}, z \in \stackrel{\circ}{\Delta}_{f}(\epsilon)
$$

where $\Gamma_{f}(\epsilon)$ (for $\|\epsilon\|$ small and outside a negligible set) is the Shilov boundary (conveniently oriented) of the connected analytic polyedrum $\Delta_{f}(\epsilon)=\left\{\left|f_{1}(\zeta)\right| \leq\right.$ $\left.\epsilon_{1}, \ldots,\left|f_{n}(\zeta)\right| \leq \epsilon_{n}\right\}$ that contains the origin (here we take representatives for the germs $f_{j}$ ). We deduce (2.9) from (2.10) just expanding the integral kernel as a geometric power series. When expressed as (2.9), the Cauchy-Weil's formula has an algebraic counterpart. For a complete presentation of it, as well as for applications, we refer to $[\mathrm{BoH} 2]$. The algebraic version of the Cauchy-Weil formula is related to a generalization of the Transformation Law that was originally proposed by Kytmanov and later generalized to the algebraic setting [BY8,BY9,BoH1].

Proposition 2.1. Let $f=\left(f_{1}, \ldots, f_{n}\right)$ and $g=\left(g_{1}, \ldots, g_{n}\right)$ be two quasiregular sequences in $\mathcal{R}$, such that the quotients $\mathcal{R} /(f)$ and $\mathcal{R} /(g)$ are finitely generated projective A-modules and

$$
g_{j}=\sum_{k=1}^{n} a_{j k} f_{k}, j=1, \ldots, n
$$

where the coefficients $a_{j k}$ are in $\mathcal{R}$. Then, for any $r_{0}, r_{1}, \ldots, r_{n} \in \mathcal{R}$, any $q \in \mathbf{N}^{n}$, we have

$$
\operatorname{Res}\left[\begin{array}{c}
r_{0} \bigwedge_{j=1}^{n} d r_{j} \\
f^{q+\underline{1}}
\end{array}\right]=\sum_{\substack{\left|q_{j}\right|=q_{j} \\
1 \leq j \leq n}} \prod_{i=1}^{n}\left(\begin{array}{c}
\mu_{i} \\
q_{i ;}
\end{array}\right) \operatorname{Res}\left[\begin{array}{c}
r_{0} \operatorname{det}\left[a_{j k}\right] \prod_{1 \leq i, j \leq n}\left(a_{i j}\right)^{q_{i, j}} \bigwedge_{j=1}^{n} d r_{j} \\
g_{1}^{\mu_{1}+1}, \ldots, g_{n}^{\mu_{n}+1}
\end{array}\right],
$$

where we have introduced the following notations for the vector $q=\left(q_{1}, \ldots, q_{n}\right)$ and the matrix of indices $q_{i, j} \in \mathbf{N}$

$q+\underline{1}=\left(q_{1}+1, \ldots, q_{n}+1\right), \quad q_{; j}=\left(q_{1, j}, \ldots, q_{n, j}\right), \quad q_{i ;}=\left(q_{i, 1}, \ldots, q_{i, n}\right), \quad \mu_{i}=\left|q_{i ;}\right|$

and

$$
\left(\begin{array}{c}
\mu_{i} \\
q_{i}
\end{array}\right)=\frac{\mu_{i} !}{q_{i, 1} ! \cdots q_{i, n} !}
$$


For our purpose, we will need here only a particular case of such an extension of the Cauchy-Weil's formula. Suppose that A is a Noetherian commutative ring and that $P_{1}, \ldots, P_{n}$ are elements in $\mathcal{R}=\mathbf{A}\left[x_{1}, \ldots, x_{n}\right]$ such that $\left(P_{1}, \ldots, P_{n}\right)$ is quasi-regular, $\mathcal{R} /\left(P_{1}, \ldots, P_{n}\right)$ is a projective $\mathbf{A}$-module and the polynomial ring $\mathbf{A}\left[x_{1}, \ldots, x_{n}\right]$ is a finetely generated $\mathbf{A}\left[P_{1}, \ldots, P_{n}\right]$ module. Then, if the $g_{j k}$ in $\mathcal{R} \otimes_{\mathbf{A}} \mathcal{R}$ are defined as

$$
1 \otimes P_{j}-P_{j} \otimes 1=\sum_{k=1}^{n} g_{j k}\left(1 \otimes x_{k}-x_{k} \otimes 1\right)
$$

then, for any $Q \in \mathcal{R}$, the Weil's formula truncates and one has a polynomial identity in $\mathcal{R}$

$$
Q(y)=\sum_{\substack{q=\in \mathbf{N}^{n} \\
|q| \leq N(\operatorname{deg} Q)}} \operatorname{Res}\left[\begin{array}{c}
Q(x) \operatorname{det}\left[g_{j k}(x, y)\right] d x_{1} \wedge \cdots \wedge d x_{n} \\
P_{1}^{q_{1}+1}(x), \ldots, P_{n}^{q_{n}+1}(x)
\end{array}\right] P_{1}^{q_{1}}(y) \cdots P_{n}^{q_{n}}(y),
$$

where $|q|=q_{1}+\cdots+q_{n}$ and $N(\operatorname{deg} Q)$ is an integer depending as an affine function on the degree of $Q$.

We will focuse on the interpretation of (2.11) in the case where $\mathbf{A}$ is a commutative field $\mathbf{K}$, which is algebraically closed and equipped with an absolute value $|\cdot|$. In this case, the fact that $\mathbf{K}\left[x_{1}, \ldots, x_{n}\right]$ is a finitely generated module over $\mathbf{K}\left[P_{1}, \ldots, P_{n}\right]$ is equivalent to the existence of constants $K \geq 0,0<\delta_{j} \leq \operatorname{deg} P_{j}$, $j=1, \ldots, n$, such that

$$
\max _{1 \leq j \leq n} \frac{\left|P_{j}(x)\right|}{|x|^{\delta_{j}}} \geq c,|x| \geq K
$$

(in other words $\left(P_{1}, \ldots, P_{n}\right)$ is a proper map with a multi-valued Lojasiewicz exponent $\left.\left(\delta_{1}, \ldots, \delta_{n}\right)\right)$. This multi-variate exponent is crucial since it controls the function $N$ that appears in formula (2.11).

The easiest case is the case when $\delta_{j}=\operatorname{deg} P_{j}, j=1, \ldots, n$. In such a case, the homogeneous polynomials $\mathcal{P}_{1}, \ldots \mathcal{P}_{n}$ obtained from the $P_{j}$ define a quasi-regular sequence in $\mathbf{K}\left[x_{0}, \ldots, x_{n}\right]$ and it follows from the Hilbert's Nullstellensatz that there exist homogeneous polynomials $\mathcal{R}_{j}\left(x_{0}, x_{j}\right), 1 \leq j \leq n$, distinguished in $x_{j}$, and homogeneous polynomials $\mathcal{R}_{j k}$ such that

$$
\mathcal{R}_{j}\left(x_{0}, x_{j}\right)=\sum_{k=1}^{n} \mathcal{R}_{j k}\left(x_{0}, \ldots, x_{n}\right) \mathcal{P}_{k}\left(x_{0}, \ldots, x_{n}\right), j=1, \ldots, n
$$

with

$$
\operatorname{deg} \mathcal{R}_{j k} \mathcal{P}_{k}=\operatorname{deg} \mathcal{R}_{j}, 1 \leq j, k \leq n
$$

Thus, we have

$$
\mathcal{R}_{j}\left(1, x_{j}\right)=R_{j}\left(x_{j}\right)=\sum_{k=1}^{n} \mathcal{R}_{j k}(1, x) P_{k}(x)=\sum_{k=1}^{n} A_{j k}(x) P_{k}(x),
$$


with

$$
\operatorname{deg}\left(A_{j k} P_{k}\right) \leq \operatorname{deg} R_{j}, 1 \leq j, k \leq n .
$$

It follows from the Transformation Law and from the Residue Calculus in one variable that

$$
\operatorname{deg} Q \leq \sum_{j=1}^{n}\left(q_{j}+1\right) D_{j}-n-1 \Longrightarrow \operatorname{Res}\left[\begin{array}{l}
Q d x_{1} \wedge \cdots \wedge d x_{n} \\
P_{1}^{q_{1}+1}, \ldots, P_{n}^{q_{n}+1}
\end{array}\right]=0
$$

This classical result goes back to Jacobi $[\mathrm{J}]$ in the complex case and has been extended in the above algebraic context in [KK] (with a different proof). This vanishing Jacobi formula has many applications in projective geometry (CayleyBacharach theorems, inversion of the Abel transform) and we refer for example to $[\mathrm{G}, \mathrm{EGH}, \mathrm{Ku} 2]$ for a presentation of some of them. A toric version of Jacobi's vanishing theorem has been obtained by Khovanskii [Kho].

Suppose now that $P_{1}, \ldots, P_{n}$ is a proper map that satisfies (2.2) and the $\delta_{k}$ are integers; let $D_{k}=\operatorname{deg} P_{k}, k=1, \ldots, n$. Suppose that $R_{1}\left(x_{1}\right), \ldots R_{n}\left(x_{n}\right)$, with homogeneisations $\mathcal{R}_{1}\left(x_{0}, x_{1}\right), \ldots \mathcal{R}_{n}\left(x_{0}, x_{n}\right)$, are in the ideal generated by $P_{1}, \ldots, P_{n}$ in $\mathbf{K}\left[x_{1}, \ldots, x_{n}\right]$ (there are still such polynomials because of the Hilbert's Nullstellensatz). One can replace condition (2.13) by the following: for any $M$ such that $M \geq \operatorname{deg} P_{j}-\delta_{j}, j=1, \ldots, n$, one has

$$
x_{0}^{M} \mathcal{R}_{j}\left(x_{0}, x_{j}\right) \in \overline{\left(x_{0}^{M+\delta_{1}-D_{1}} \mathcal{P}_{1}, \ldots, x_{0}^{M+\delta_{n}-D_{n}} \mathcal{P}_{n}\right)}
$$

where the integral closure is taken in the ring $\mathbf{K}\left[\left[x_{0}, \ldots, x_{n}\right]\right]$. If one uses the theorem of Lipman-Teissier [LS, LT], one obtains, for any $\lambda \in \mathbf{N}^{*}$,

$$
\left(x_{0}^{M} \mathcal{R}_{j}\left(x_{0}, x_{j}\right)\right)^{\lambda+n} \in\left(x_{0}^{M+\delta_{1}-D_{1}} \mathcal{P}_{1}, \ldots, x_{0}^{M+\delta_{n}-D_{n}} \mathcal{P}_{n}\right)^{\lambda+1} .
$$

Therefore, one can write, for any such $\lambda$,

$$
R_{j}\left(x_{j}\right)^{\lambda+n}=\sum_{\substack{l=\left(l_{1}, \ldots, l_{n}\right) \\ l_{1}+\cdots+l_{n}=\lambda+1}} U_{j l}^{(\lambda)}(x) P_{1}^{l_{1}}(x) \cdots P_{n}^{l_{n}}(x), j=1, \ldots, n
$$

with

$\operatorname{deg} U_{j l}^{(\lambda)} \leq(n-1) M+(\lambda+n) \operatorname{deg} R_{j}-\sum_{k=1}^{n} \delta_{k} l_{k}, l \in \mathbf{N}^{n},|l|=\lambda+1, j=1, \ldots, n$.

For example, if we assume that all $D_{j}=D$, all $\delta_{j}=\delta$ (then one takes $M=D-\delta$ ), we obtain that for any multiindex $q=\left(q_{1}, \ldots, q_{n}\right) \in \mathbf{N}^{n}$,

$$
R_{j}\left(x_{j}\right)^{|q|+n}=\sum_{k=1}^{n} A_{j k}^{(q)} P_{k}^{q_{k}+1}
$$

with

$$
\operatorname{deg} A_{j k}^{(q)} P_{k}^{q_{k}+1} \leq(|q|+n)\left(D-\delta+\operatorname{deg} R_{j}\right), 1 \leq j, k \leq n .
$$


If follows again from the Transformation Law and from Residue Calculus in one variable that, for any $Q \in \mathbf{K}\left[x_{1}, \ldots, x_{n}\right]$,

$$
\operatorname{deg} Q+(|q|+n)((n-1) D-n \delta)) \leq-n-1 \Longrightarrow \operatorname{Res}\left[\begin{array}{c}
Q d x_{1} \wedge \cdots \wedge d x_{n} \\
P_{1}^{q_{1}+1}, \ldots, P_{n}^{q_{n}+1}
\end{array}\right]=0
$$

This shows that if $1-\frac{1}{n}<\frac{\delta}{D} \leq 1$, one can express the function $N$ in $(2.11)$ in terms of the Lojasiewicz exponent $\delta$. In fact, one may conjecture that one has the following version of Jacobi's vanishing theorem

$$
\operatorname{deg} Q \leq \sum_{j=1}^{n}\left(q_{j}+1\right) \delta_{j}-n-1 \Longrightarrow \operatorname{Res}\left[\begin{array}{l}
Q d x_{1} \wedge \cdots \wedge d x_{n} \\
P_{1}^{q_{1}+1}, \ldots, P_{n}^{q_{n}+1}
\end{array}\right]=0
$$

Here again, the validity of the result in the complex case may give some insight into the validity of the above conjecture.

Proposition 2.2. Let $P_{1}, \ldots, P_{n}, n$ polynomials in $\mathbf{C}\left[x_{1}, \ldots, x_{n}\right]$ such that there exists $\delta_{1}, \ldots, \delta_{n}$, with $0<\delta_{j} \leq \operatorname{deg} P_{j}, K \geq 0, c>0$, such that

$$
\|x\| \geq K \Longrightarrow \max _{1 \leq j \leq n} \frac{\left|P_{j}(x)\right|}{|x|^{\delta_{j}}} \geq c,
$$

Then, one has, for any polynomial $Q \in \mathbf{C}\left[x_{1}, \ldots, x_{n}\right]$,

$$
\operatorname{deg} Q \leq \sum_{j=1}^{n}\left(q_{j}+1\right) \delta_{j}-n-1 \Longrightarrow \operatorname{Res}\left[\begin{array}{l}
Q d x_{1} \wedge \cdots \wedge d x_{n} \\
P_{1}^{q_{1}+1}, \ldots, P_{n}^{q_{n}+1}
\end{array}\right]=0
$$

Sketch of proof. It is enough to prove the statement when $q=0$. Moreover, using changes of basis of the form $x_{1}=y_{1}^{M}, \ldots, x_{n}=y_{n}^{M}$ and the compatibility of Residue Calculus with change of basis [L], one can assume that the $\delta_{j}$ are integers. The key idea is just to choose $M$ as before and to set

$$
s_{j}(\zeta)=\frac{1}{(1+\|\zeta\|)^{M+\delta_{j}}} \overline{P_{j}}, j=1, \ldots, n
$$

We also define

$$
\|P\|_{\delta}^{2}=\sum_{k=1}^{n} \frac{\left|P_{k}\right|^{2}}{\left(1+\|\zeta\|^{2}\right)^{\delta_{k}+M}} .
$$

Using the Bochner-Martinelli formulas, for $R$ sufficiently big one has

$$
\operatorname{Res}\left[\begin{array}{c}
Q d x \\
P
\end{array}\right]=\gamma_{n}\left[\int_{\|\zeta\|=R}\|P\|_{\delta}^{2(\lambda-n)} Q\left(\sum_{k=1}^{n}(-1)^{k-1} s_{k} d s_{[k]}\right) \wedge d \zeta\right]_{\lambda=0}
$$

where the right-hand side can be understood as the value at $\lambda=0$ of an entire function of $\lambda$. We now express the differential form

$$
\|P\|_{\delta}^{2(\lambda-n)} Q\left(\sum_{k=1}^{n}(-1)^{k-1} s_{k} d s_{[k]}\right) \wedge d \zeta
$$


in homogeneous coordinates. We denote this homogeneous form $\Omega_{Q}(\lambda ; \tilde{\zeta})$, where $\tilde{\zeta}=\left(\zeta_{0}, \ldots, \zeta_{n}\right)$, and we rewrite $(2.17)$ as

$$
\operatorname{Res}\left[\begin{array}{c}
Q d x \\
P
\end{array}\right]=-\gamma_{n}\left[\int_{\Sigma} \bar{\partial} \Omega_{Q}(\lambda ; \tilde{\zeta})\right]_{\lambda=0},
$$

where $\Sigma$ is the complement (in $\mathbf{P}^{n}$ ) of a finite union of balls centered at the common zeroes (in the affine space) of the polynomials $P_{j}, 1 \leq j \leq n$. We now compute the meromorphic continuation of the right hand side of (2.18) using a resolution of singularities for the hypersurface $\zeta_{0} \mathcal{P}_{1} \cdots \mathcal{P}_{n}=0$ near any point $\xi$ of $\mathbf{P}^{n}$ on the hyperplane at infinity $\zeta_{0}=0$, and then a normalization. A careful study of all terms show that when $\operatorname{deg} Q \leq \delta_{1}+\ldots+\delta_{n}-n-1$, or more precisely when $n M+\delta_{1}+\ldots+\delta_{n}-n-1-\operatorname{deg} Q \geq n M$, one has

$$
\left[\int_{\Sigma} \bar{\partial} \Omega(\lambda ; \tilde{\zeta})\right]_{\lambda=0}=0
$$

This is due to the fact that $\zeta_{0}^{n M+|\delta|-n-1}$ appears in the numerator of $\bar{\partial} \Omega_{Q}(\lambda ; \tilde{\zeta})$ while the currents involved in $\bar{\partial} \Omega_{Q}(0 ; \tilde{\zeta})$ are annihilated by holomorphic functions that lie in the $n$-th power of the integral closure of the ideal generated by the $\zeta_{0}^{M+\delta_{j}-D_{j}} \mathcal{P}_{j}$ in $\mathcal{O}_{\xi}$; from $(2.2)$ we conclude that the germ at $\xi$ of $\zeta_{0}^{M}$ is in this integral closure, therefore the germ at $\xi$ of $\zeta_{0}^{n M}$ annihilates the currents involved in $\Omega_{Q}(0 ; \tilde{\zeta})$. As one can see, such a proof is directly inspired from the analytic techniques we introduced to study problems related to the Fundamental Principle. $\diamond$

Coming back to the algebraic setting, we have already at our disposal the following very useful proposition, which is a different version of the classical DivisionInterpolation formula of Kronecker

Proposition 2.3. Let $P_{1}, \ldots, P_{n}$ be $n$ polynomials with degree $D$ in $\mathbf{K}\left[x_{1}, \ldots, x_{n}\right]$ such that

$$
|x| \geq K \Longrightarrow \max _{1 \leq j \leq n} \frac{\left|P_{j}(x)\right|}{\|x\|^{\delta}} \geq c>0
$$

for some integer $\delta$ in $] 0, D]$. Suppose that $1-\frac{1}{n(n+1)}<\frac{\delta}{D} \leq 1$. Suppose that

$$
1 \otimes P_{j}-P_{j} \otimes 1=\sum_{k=1}^{n} g_{j k}\left(1 \otimes x_{k}-x_{k} \otimes 1\right), j=1, \ldots, n .
$$

Then one has

$$
1=\operatorname{Res}\left[\begin{array}{c}
\operatorname{det}\left[g_{j k}(x, y)\right] d x_{1} \wedge \cdots \wedge d x_{n} \\
P_{1}(x), \ldots, P_{n}(x)
\end{array}\right]
$$

Proof. We already know that

$$
\operatorname{deg} Q+(|q|+n)((n-1) D-n \delta) \leq-n-1 \Longrightarrow \operatorname{Res}\left[\begin{array}{c}
Q d x_{1} \wedge \cdots \wedge d x_{n} \\
P_{1}^{q_{1}+1}, \ldots, P_{n}^{q_{n}+1}
\end{array}\right]=0
$$


We just need to check that the inequality

$$
n(D-1)+(n+1)((n-1) D-n \delta) \leq-n-1
$$

is satisfied. This means

$$
n D+(n+1)((n-1) D-n \delta)<0
$$

that is

$$
\left(n^{2}+n-1\right) D-n(n+1) \delta<0,
$$

which is the condition required. $\diamond$

Remark. In the complex case, where the $P_{j}$ satisfy the condition $(2.2)$ and

$$
\operatorname{det}\left[g_{j k}\right](x, y)=\sum_{|\alpha|+|\beta| \leq|D|-n} \gamma_{\alpha \beta} x^{\alpha} y^{\beta}
$$

(where $|D|=D_{1}+\ldots D_{n}$ ), the general Division-Interpolation formula of Kronecker can be stated as follows. For any $Q \in \mathbf{K}\left[x_{1}, \ldots, x_{n}\right]$,

$$
Q(y)=\sum_{\substack{q, \alpha, \beta \in \mathbb{N}^{n} \\
|\alpha|+|\beta| \leq|D|-n \\
<q+1, \delta>+|\beta| \leq|D|+\operatorname{deg} Q}} \gamma_{\alpha \beta} \operatorname{Res}\left[\begin{array}{c}
Q x^{\alpha} d x_{1} \wedge \cdots \wedge d x_{n} \\
P_{1}^{q_{1}+1}, \ldots, P_{n}^{q_{n}+1}
\end{array}\right] y^{\beta} P_{1}^{q_{1}}(y) \cdots P_{n}^{q_{n}}(y) .
$$

This follows immediately from the Cauchy-Weil formula, together with Proposition 2.2. Such a formula probably holds in the general algebraic case where $\mathbf{C}$ is replaced by a field $\mathbf{K}$ with arbitrary caracteristic. Note that if $Q$ is in the ideal generated by $P_{1}, \ldots, P_{n},(2.20)$ gives an explicit formula

$$
Q=\sum_{k=1}^{n} A_{k} P_{k}
$$

with very precise estimates on the degrees of the $A_{k}$ in terms of the degree of $Q$ and of the Lojasiewicz multi-exponent $\left(\delta_{1}, \ldots, \delta_{n}\right)$.

Proposition 2.3 is the major step in our direct formulation of the algebraic Nullstellensatz over an integral domain $\mathbf{A}$ whose quotient field $\mathbf{K}$ is an infinite field with arbitrary caracteristic. We will express here a Bézout identity, in the same vein than the Bézout identities one can write in the Paley-Wiener algebra for inconsistent systems of convolution equations which satisfy the Fundamental Principle [BY3]. We start with a collection of polynomials $P_{1}, \ldots, P_{m}$ in $\mathbf{A}\left[x_{1}, \ldots, x_{n}\right]$ without common zeroes in $\overline{\mathbf{K}}^{n}$. Let $d=D_{1} \ldots D_{\mu}$, where $\mu=\inf (n, m)$ and the degrees are in decreasing order. Then one can find linear combinations

$$
p_{j}=\sum_{k=1}^{m} \lambda_{j k} P_{k}, j=1, \ldots, n, \lambda_{j k} \in \mathbf{A}
$$

such that $\operatorname{deg} p_{j}=D_{1}, 1 \leq j \leq n$ and any subfamily of $\left\{p_{1}, \ldots, p_{n}\right\}$ defines a quasiregular sequence in $\mathbf{K}\left[x_{1}, \ldots, x_{n}\right]$. If one uses the Ji-Kollár-Shiffman Lojasiewicz 
inequalities $[\mathrm{JKS}]$ and the Normalization Theorem of E. Noether, we can assume, after a linear change of variables over $\mathbf{A}$, that, for any integer $l \geq 2$, the map

$$
x \mapsto\left(x_{1}^{l d} p_{1}(x), \ldots, x_{n}^{l d} p_{n}(x)\right)
$$

is proper, with multivalued Lojasiewicz exponent $(l d-d, \ldots, l d-l)$. Then, if $l$ is sufficiently large so that

$$
1-\frac{1}{n(n+1)} \leq \frac{d(l-1)}{l d+D_{1}} \leq 1
$$

we can apply Proposition 2.3 and obtain the Kronecker identity

$$
1=\operatorname{Res}\left[\begin{array}{c}
\operatorname{det}\left[g_{j k}^{(l)}(x, y)\right] d x_{1} \wedge \cdots \wedge d x_{n} \\
x_{1}^{l d} p_{1}(x), \ldots, x_{n}^{l d} p_{n}(x)
\end{array}\right]
$$

where

$$
1 \otimes x_{j}^{l d} p_{j}-x_{j}^{l d} p_{j} \otimes 1=\sum_{k=1}^{n} g_{j k}^{(l)}\left(1 \otimes x_{k}-x_{k} \otimes 1\right) .
$$

There exists a linear combination $p_{0}$, with coefficients in $\mathbf{R}$, of the $P_{j}$, which does not vanish on the common zero set of the polynomials $x_{j} p_{j}(x)$. There are $g_{0 j} \in \mathbf{A}\left[x_{1}, \ldots, x_{n}\right] \otimes_{\mathbf{A}} \mathbf{A}\left[x_{1}, \ldots, x_{n}\right]$ such that

$$
1 \otimes p_{0}-p_{0} \otimes 1=\sum_{k=1}^{n} g_{0 k}\left(1 \otimes x_{k}-x_{k} \otimes 1\right)
$$

When $\mathcal{R}=\mathbf{K}\left[x_{1}, \ldots, x_{n}\right]$, and $\left(f_{1}, \ldots, f_{n}\right)$ is a quasi-regular sequence in this algebra, it is immediate to extend the definition of the residue symbol

$$
\operatorname{Res}\left[\begin{array}{c}
r_{0} d x_{1} \wedge \cdots \wedge d x_{n} \\
f_{1}, \ldots, f_{n}
\end{array}\right], r_{0} \in \mathcal{R}
$$

to the case where $r_{0}$ is an element of $\mathbf{K}\left(x_{1}, \ldots, x_{n}\right)$ which has no poles at the common zeros of $f_{1}, \ldots, f_{n}$. Therefore, one can rewrite $(2.21)$ as

$$
1=\sum_{j=1}^{n} \operatorname{Res}\left[\begin{array}{c}
\frac{\Delta_{j}^{(l)}(x, y)}{p_{0}(x)} d x \\
x_{1}^{l d} p_{1}, \ldots, x_{n}^{l d} p_{n}
\end{array}\right] y_{j}^{l d} p_{j}(y)+\operatorname{Res}\left[\begin{array}{c}
\frac{\operatorname{det}\left[g_{j k}^{(l)}(x, y)\right]}{p_{0}(x)} d x \\
x_{1}^{l d} p_{1}, \ldots, x_{n}^{l d} p_{n}
\end{array}\right] p_{0}(y)
$$

where $d x=d x_{1} \wedge \cdots \wedge d x_{n}$ and $\Delta_{j}^{(l)}(x, y)$ is obtained from $\operatorname{det}\left[g_{j k}^{(l)}(x, y)\right]$ by substitution of the column of index $j$ by the column-vector $\left(g_{01}(x, y), \ldots, g_{0 n}(x, y)\right)$. This formula $(2.22)$ can be rewritten as the Bézout identity in $\mathbf{K}\left[x_{1}, \ldots, x_{n}\right]$

$$
1=\sum_{k=1}^{m} A_{k} P_{k}, \operatorname{deg} A_{k} P_{k} \leq(n+1)\left(l d+D_{1}\right)-n .
$$


Eliminating the denominators in such a formula provides the identity

$$
\begin{gathered}
a=\sum_{k=1}^{m} U_{k} P_{k} \\
a \in \mathbf{A}^{*}, U_{k} \in \mathbf{A}\left[x_{1}, \ldots, x_{n}\right], \operatorname{deg} U_{k} P_{k} \leq(n+1)\left(l d+D_{1}\right)-n .
\end{gathered}
$$

It remains to study carefully the size estimates in such an identity when one makes the assumption that the integral domain $\mathbf{A}$ is a regular factorial ring equipped with a size.

\section{Complexity of the Residue Calculus}

Let $\mathbf{A}$ be a regular factorial domain with quotient field $\mathbf{K}$, such that the ring of polynomials in an arbitrary number of variables with coefficients in $\mathbf{A}$, $\operatorname{Pol}(\mathbf{A})$, is equipped with a logarithmic size. We have already mentioned the case of $\operatorname{Pol}(\mathbf{Z})$ with the Mahler measure. We can also mention $\operatorname{Pol}\left(\mathbf{F}_{p}\left[\tau_{1}, \ldots, \tau_{q}\right]\right)$ with the degee in $\tau$ as a size. Let $P_{1}, \ldots, P_{n}$ be polynomials in $\mathbf{A}\left[x_{1}, \ldots, x_{n}\right]$ defining a quasiregular sequence in $\mathbf{K}\left[x_{1}, \ldots, x_{n}\right]$. The explicit versions of the Bézout identity we proposed in the last section involve computing residue symbols of the form

$$
\operatorname{Res}\left[\begin{array}{c}
\frac{Q_{1}}{Q_{2}} d x_{1} \wedge \cdots \wedge d x_{n} \\
P_{1}^{q_{1}+1}, \ldots, P_{n}^{q_{n}+1}
\end{array}\right], q \in \mathbf{N}^{n},
$$

where $Q_{1}, Q_{2} \in \mathbf{A}\left[x_{1}, \ldots, x_{n}\right]$, and $\left(P_{1}, \ldots, P_{n}, Q_{2}\right)$ generate $\mathbf{K}\left[x_{1}, \ldots, x_{n}\right]$ in this ring. Let $D_{j}=\operatorname{deg} P_{j}, 1 \leq j \leq n$. The key idea that one can use in order to compute symbols of the form (3.1) is to find first a system of relations of integral dependency

$$
\mathcal{Q}_{j}\left(x_{j}, P\right)=\sum_{k=0}^{N_{j}} A_{j k}(P) x_{j}^{N_{j}-k}=0, j=1, \ldots, n,
$$

where the $A_{j k}$ are polynomials in $y$ with coefficients in A. An old theorem of $\mathrm{O}$. Perron [Per] implies that it is possible to find such relations at the same time that the following estimate holds,

$$
\operatorname{deg}_{y} \mathcal{Q}_{j}\left(y_{0}, y_{1}^{D_{1}}, \ldots, y_{n}^{D_{n}}\right) \leq D_{1} \cdots D_{n} .
$$

Unfortunately, the size estimates that one can predict for the coefficients of the $A_{j l}$ are just the ones that can be predicted with plain linear algebra are are roughly in $D^{n^{2}} h$, where $D$ is the maximum of the $D_{j}$ and $h$ the maximum of the logarithmic sizes of the $P_{j}$. There are at several ways to bypass this problem:

- Use a deep result of P. Philippon [Phi] about estimates for denominators in the Hilbert Nullstellensatz. Such estimates follow once again from the application of Lipman-Teissier's theorem. We use them here for the polynomials $x_{j}-u_{0}, P_{1}(x)-u_{1}, \ldots, P_{n}(x)-u_{n}$ considered as polynomials in $\mathbf{A}\left[u_{0}, u_{1}, \ldots, u_{n}\right]$ without common zeroes in $\mathbf{K}\left(u_{0}, \ldots, u_{n}\right)$. This was done for example in [BY9]. The size estimates are in $\kappa(n) D_{1} \cdots D_{n}(h+D)$. 
- Notice, as in [EIM2], that one can obtain a relation of the form (3.2) from a maximal non identically zero minor of the Bezoutian matrix of the polynomials $x_{j}-u_{0}, P_{1}-u_{1}, \ldots, P_{n}-u_{n}$. The degree estimates are worse than in Perron's result, but the size estimates are also in $\kappa(n) D^{O(n)} h$.

- Introduce a perturbation parameter $\lambda$ and construct auxiliary relations

$$
\sum_{k=0}^{N_{j}} \widetilde{A}_{j k}\left(\lambda, \lambda x_{1}^{D_{1}+1}+P_{1}, \ldots, \lambda x_{n}^{D_{n}+1}+P_{n}\right) x_{j}^{N_{j}-k}=0, j=1, \ldots, n,
$$

using the fact that residue calculus is quite easy to perform if the entries $P_{j}$ are a Gröbner Basis for some weight [CDS]. Here again, the size estimates are of the same type as above. Such an idea comes from Analysis, see for example [AY].

In any case, one can rewrite these relations (3.2) or (3.3) as

$$
\mathcal{Q}_{j}\left(x_{j}, u\right)=\sum_{k=1}^{n} Q_{j k}\left(x_{j}, P, u\right)\left(P_{j}-u\right)
$$

or

$$
\widetilde{\mathcal{Q}}_{j}\left(x_{j}, u, \lambda\right)=\sum_{k=1}^{n} \widetilde{A}_{j k}(x, P, u, \lambda)\left(P_{j}-u+\lambda x_{j}^{D_{j}+1}\right)
$$

We introduce generic parameters $\alpha \in \mathbf{A}^{n}$ or $\tilde{\alpha} \in \mathbf{A}^{n+1}$ (the quotient field $\mathbf{K}$ is assumed to be infinite) in order to rewrite (3.4) or (3.5) as

$$
\mathcal{Q}_{j}\left(x_{j}, t \alpha\right)=\sum_{k=1}^{n} Q_{j k}\left(x_{j}, P, t \alpha\right)\left(P_{j}-\alpha_{j} t\right)=t^{\nu_{j}}\left(R_{j}\left(\alpha, x_{j}\right)+t S_{j}\left(\alpha, t, x_{j}\right)\right)
$$

or

$$
\begin{aligned}
\widetilde{\mathcal{Q}}_{j}\left(x_{j}, t \tilde{\alpha}\right) & =\sum_{k=1}^{n} \widetilde{A}_{j k}(x, P, t \tilde{\alpha})\left(P_{j}-t\left(\tilde{\alpha}_{j}-\tilde{\alpha}_{n+1} x_{j}^{D_{j}+1}\right)\right) \\
& =t^{\nu_{j}}\left(\widetilde{R}_{j}\left(\tilde{\alpha}, x_{j}\right)+t \widetilde{S}_{j}\left(\tilde{\alpha}, t, x_{j}\right)\right) .
\end{aligned}
$$

Using the generalized transformation law (2.8), one obtains the calculus of the residue symbols

$$
\operatorname{Res}\left[\begin{array}{c}
\frac{Q_{1}(x)}{Q_{2}(x)} d t \wedge d x \\
t^{q_{0}+1}, P_{1}-\alpha_{1} t, \ldots, P_{n}-\alpha_{n} t
\end{array}\right]=\sum_{|q|=q_{0}} \alpha^{q} \operatorname{Res}\left[\begin{array}{c}
\frac{Q_{1}}{Q_{2}} d x \\
P_{1}^{q_{1}+1}, \ldots, P_{n}^{q_{n}+1}
\end{array}\right]
$$

for $q_{0} \in \mathbf{N}$, or

$$
\operatorname{Res}\left[\begin{array}{c}
\frac{Q_{1}(x)}{Q_{2}(x)} d t \wedge d x \\
t, P_{1}-t\left(\tilde{\alpha}_{1}-\tilde{\alpha}_{n+1} x_{1}^{D_{1}+1}\right), \ldots, P_{n}-t\left(\tilde{\alpha}_{n}-\tilde{\alpha}_{n+1} x_{n}^{D_{n}+1}\right)
\end{array}\right]=\operatorname{Res}\left[\begin{array}{c}
\frac{Q_{1}}{Q_{2}} d x \\
P
\end{array}\right] .
$$

Once the calculus of residue symbols is performed in that way, one can show that the solution of the Bézout identity (2.23) satisfies size estimates of the form

$$
\max \left(h(a), \max _{k} h\left(A_{k}\right)\right) \leq \kappa(n)\left(D_{1} \ldots D_{n}\right)^{4} D_{1}^{2}(h+\log m+D)
$$


where $D_{1} \geq D_{2} \ldots \geq D_{m}$ (we assume $m \geq n$, which is always possible if we allow repetitions).

One could hope that the use of the Cauchy-Weil's formula over a ring (and not a field as here), as introduced in [BoY2], may provide a better understanding of such an effective method for the solution of the arithmetic Nullstellensatz. Let us mention here that in [BY7], we developped analytic methods to compute explicitely Green currents and arithmetic height of cycles in $\mathcal{X}=\operatorname{Proj} \mathbf{Z}\left[x_{0}, \ldots, x_{n}\right]$ (that are defined as complete intersections in $\mathcal{X}(\mathbf{C})=\mathbf{P}^{n}$ ) as residues at 0 of zetafunctions. The resolution of the arithmetic Nullstellensatz we proposed in these two sections, as the construction of Green currents using analytic continuation techniques, should fit in a natural way with multivariate Arakelov theory.

\section{Conclusion.}

As we have seen in this paper, the methods originally introduced to solve questions related to the Fundamental Principle or the conjecture of L. Ehrenpreis appear to be quite useful in order to find effective solutions to some problems in Algebraic Geometry . It is also clear that one should profit from the ideas developped by algebraists or geometers and adapt them to an analytic context. Such is the case for example for the techniques involved in the study of sparse problems in commutative algebra or algebraic geometry (sparse resultant, toric varieties, toric residues, geometric description of amoebas,...). Some of the tools presented here can be extended to the analytic context and to the study of exponential polynomials in several variables initiated by Polya, and pursued by Khovanskii, Kazarnovski, Ronkin, could be obtained using these tools. For example, in [Pel] it is proved (under convenient arithmetic conditions) that the Bézout identity where the entries are sums of exponentials can be solved using the Division-Interpolation methods based on the residue calculus that we developped in the second section.

\section{References}

[AY] L. A. Aizenberg, A. P. Yuzhakov, Integral Representations and Residues in Multidimensional Complex Analysis, Transl. Math. Monographs 58, AMS, 1983. [Ba] A. Baker, Transcendental Number Theory, Cambridge University Press, London, 1979 .

[BGS] J. B. Bost, H. Gillet, and C. Soulé, Heights of projective varieties and positive Green forms, J. Amer. Math. Soc. 7 (1994), 903-1027.

[BGVY] C. A. Berenstein, R. Gay, A. Vidras, and A. Yger, Residue currents and Bézout identities, Progress in Mathematics 114, Birkhäuser, Basel-Boston-Berlin, 1993.

[Bjo] J.-E. Björk, Rings of Differential Operators, North-Holland Publ. Co., Amsterdam, 1979.

[BoH1] J. Y. Boyer, M. Hickel, Une généralisation de la loi de transformation pour les résidus, Bull. Soc. Math. France 125, 1997, 315-335.

[BoH2] J. Y. Boyer, M. Hickel, Extension dans un cadre algébrique d'une formule de Weil, Prépublication 27, Bordeaux 1, 1996.

[BS] J. Briançon and H. Skoda, Sur la clôture intégrale d'un idéal de germes de fonctions holomorphes en un point de $\mathbf{C}^{n}$, Comptes Rendus Acad. Sci. Paris, série A, 278 (1974), 949-951. 
[BT] C. A. Berenstein and B. A. Taylor, Interpolation problems in $\mathbf{C}^{n}$ with applications to harmonic analysis, J. Analyse Math. 38 (1980), 188-254.

[BTY] C.A. Berenstein, B.A. Taylor and A. Yger, Sur les systèmes d'équations différence-différentelles, Ann. Inst. Fourier 33 (1983), 109-130.

[BY1] C.A. Berenstein and A. Yger, Ideals generated by exponential polynomials, Advances in Math. 60 (1986), 1-80.

[BY2] C.A. Berenstein and A. Yger, On Lojasiewicz type inequalities for exponential polynomials, J. Math. Anal. Appl. 129 (1988), 166-195.

[BY3] C.A. Berenstein and A. Yger, Analytic Bezout identities, Advances in Applied Math. 10 (1989), 51-74.

[BY4] C. A. Berenstein and A. Yger, Effective Bézout identities in $Q\left[z_{1}, \ldots, z_{n}\right]$, Acta Math. 166 (1991), 69-120.

[BY5] C. A. Berenstein and A. Yger, Une formule de Jacobi et ses conséquences, Ann. Sci. Ecole Norm. Sup. 24 (1991), 363-377.

[BY6] C. A. Berenstein and A. Yger, Exponential polynomials and $\mathcal{D}$ modules, Compositio Mathematica 95, 1995, 131-181.

[BY7] C. A. Berenstein and A. Yger, Green currents and analytic continuation, to appear in J. Analyse. Math.

[BY8] C. A. Berenstein and A. Yger, Residues and effective Nullstellensatz, Electronic Research Announcements of the Amer. Math. Soc. 2 (1996), 82-91.

[BY9] C. A. Berenstein and A. Yger, Residue calculus and effective Nullstellensatz, ISR Technical Report 96.

[CDS] E. Cattani, A. Dickenstein, B. Sturmfelds, Computing multidimensional residues, in Algorithms in Algebraic Geometry and Applications, Prog. in Math 143, Birkhäuser, 1996.

$[\mathrm{CH}]$ N. Coleff and M. Herrera, Les courants résidus associés à une forme méromorphe, Springer LN 633, 1978.

[EGH] D. Eisenbud, M. Green, and J. Harris, Cayley-Bacharach theorems and conjectures, Bull. Amer. Math. Soc. 33 (1996), 295-324.

[Ehr] L. Ehrenpreis, Fourier Analysis in Several Complex Variables, John Wiley \& Sons, 1970.

[ElM1] M. Elkadi and B. Mourrain, Approche algébrique des résidus algébriques, Rapport de Recherche 2884, INRIA, 1996.

[ElM2] M. Elkadi and B. Mourrain, Some applications of Bezoutians in Effective Algebraic Geometry, preprint INRIA 1998.

[G] P. Griffiths, Variations on a Theorem of Abel, Inventiones math, 35, 321 (1976), 321-390.

[Gu] D.I. Gurevich, Counterexamples to a problem of L. Schwartz, Funct. Anal. Appl., 9 (1975), 116-120.

[J] C. G. Jacobi, Theoremata nova algebraica circa systema duarum aequationum inter duas variabiles propositarum, in Gesammelte Werke, Band III, 285-294.

[JKS] S. Ji, J. Kollár and B. Shiffman, A Global Lojasiewicz Inequality for Algebraic Varieties, Trans. Amer. Math. Soc. 329 (1992), 813-818.

[Kho] A. Khovanskii, Newton polyedra and the Euler-Jacobi formula, Russian Math. Surveys 33, 1978, 237-238.

[KK] M. Kreutzer, E. Kunz, Traces in strict Frobenius algebras and strict complete intersections, J. reine. angew. Math. 381 (1987), 181-204. 
[Ko] J. Kollár, Sharp effective Nullstellensatz, J. Amer. Math. Soc 1, 4 (1988), 963-975.

[KK] M. Kreutzer, E. Kunz, Traces in strict Frobenius algebras and strict complete intersections, J. reine. angew. Math. 381 (1987), 181-204.

[Ku1] E. Kunz, Kähler differentials, Vieweg Advanced Lectures in Mathematics, Braunschweig, Wiesbaden, 1986.

[Ku2] E. Kunz, Über den $n$-dimensionalen Residuensatz, Jahresbericht der Deutschen Mathematiker-Vereinigung 94 (1992), 170-188.

[L] J. Lipman, Residues and Traces of Differential Forms via Hochschild Homology, Contemporary Mathematics 61, American Mathematical Society, Providence, 1987.

[LS] J. Lipman and A. Sathaye, Jacobian ideals and a theorem of Briançon-Skoda, Michigan Math, J. 28 (1981), 199-222.

[LT] J. Lipman and B. Teissier, Pseudo-rational local rings and a theorem of Briançon-Skoda about integral closures of ideals, Michigan Math. J. 28 (1981), 97-116.

[Pal] V. Palamodov, Linear differential operators with constant coefficients, Springer-Verlag, Berlin, 1967, Zbl. 191, 434.

[Pel] T. Pellé, Identités de Bézout pour certains systèmes de sommes d'exponentielles, Ark. Mat. 36, 1998, 1, 131-162.

[Per] O. Perron, Algebra I (Die Grundlagen) Göschens Lehrbücherei, Berlin und Leipzig, 1932.

[Phi] P. Philippon, Dénominateurs dans le théorème des zéros de Hilbert, Acta Arithm. LVIII. 1 (1991), 1-25.

[ScS] G. Scheja and U. Storch, Über Spurfunktionen bei vollständigen Durschnitten, Journal reine angew. Mathematik, 278, 174-190, 1975.

[Te1] B. Teissier, Résultats récents d'algèbre commutative effective, Séminaire Bourbaki 1989-1990, Astérisque 189-190, 107-131.

[Te2] B. Teissier, Variétés polaires II, Algebraic Geometry, La Rabida, Springer LN 961, 1980, 71-146.

Institute for Systems Research, University of Maryland, College Park, MD 20742 carlos@src.umd.edu

Lab. de Math. Pures, Université Bordeaux Sciences, 33405 Talence, France yger@math.u-bordeaux.fr 